\title{
Large-Eddy Simulation of Approaching-Flow Stratification on Dispersion over Arrays of Buildings
}

\author{
Zheng-Tong Xie* \\ Aeronautics and Astronautics, Faculty of Engineering and the Environment \\ University of Southampton, Southampton, SO17 1BJ, UK \\ Paul Hayden \\ Environmental Flow Research Laboratory, Faculty of Engineering and Physical Sciences, \\ University of Surrey, Guildford, GU2 7XH, UK \\ Curtis R. Wood ${ }^{1}$ \\ Finnish Meteorological Institute, Erik Palménin aukio 1, 00101 Helsinki, Finland.
}

\begin{abstract}
The study investigates thermal stratification effects of approach flows on dispersion in urban environments. This is in some ways analogous to a well developed non-neutral flow (e.g. through a large urban area) approaching a neighborhoodscale urban region, where the effect of the local heat transfer was assumed less important. A generic urban-type geometry, i.e. a group of staggered cubes, was taken as the first test case. The DAPPLE site, which was about a one-km ${ }^{2}$ region near the intersection of Marylebone Road and Gloucester Place in central London, was taken as the second test case. Only weakly unstable conditions (i.e. bulk Richardson number $R_{b} \geq-0.2$ ) of approach flows were considered, with adiabatic boundary conditions at the ground and building surfaces. A number of numerical experiments including with various $R_{b}$ were performed. The modelled mean concentration for $R_{b}=-0.1$ gave the best agreement with the field data at all DAPPLE stations. This suggests that stratification effects on dispersion in weakly unstable conditions (e.g. in London) are not negligible.
\end{abstract}

Keywords: thermal stability, wind variation, dispersion, urban environments

\footnotetext{
*Email: z.xie@ @oton.ac.uk

${ }^{1}$ Former institute:Department of Meteorology, University of Reading, Reading, RG6 6BB, UK
} 


\section{Introduction}

We wish to accurately model scalar dispersion over short ranges $(<1 \mathrm{~km})$ in full-scale urban environments. Large-eddy simulation (LES), a relevant promising tool, is particularly useful for modelling genuine unsteadiness of plume dispersion (Niceno and Hanjalić, 2002; Gousseau et al., 2011), e.g. plume meandering. However, some issues should be solved.

Mean concentration, from small-scale physical or numerical DAPPLE models, can be one order greater than obtained in field experiments (Cheng and Robins, 2004; Xie and Castro, 2009). The discrepancy might be attributed to: (I) variation of wind direction and magnitude because of background weather conditions; (II) thermal buoyancy effects of approach flows and local heat transfer from/to buildings; (III) small roughness elements;(IV) Reynolds-number effects and (V) effects of boundary-layer depth.

Urban dispersion is sensitive to variable wind direction (Xie, 2011). A study on (I) reported in Xie (2011) used DAPPLE wind data (Wood et al., 2009, 2010) measured on BT Tower $190 \mathrm{~m}$ above street level - turbulent eddies with scales below one minute were re-generated as in Xie and Castro (2008) - and drove LES. The approach generates turbulent inflows satisfying prescribed integral length scales and Reynolds stress-tensor. It is efficient: at every time-step only one set of two-dimensional (rather than three-dimensional) random data is filtered to generate a set of two-dimensional data with appropriate spatial correlations, and these data correlate with data from previous time-step by using an exponential function based on two weighting factors. The DAPPLE site (Wood et al., 2009) is a region, about $1 \mathrm{~km}^{2}$, near the intersection of Marylebone Road and Gloucester Place in central London (hereafter denoted as the DAPPLE intersection). When using BT Tower data to generate inlet boundary conditions to drive LES, predicted dispersion in the near field (i.e. $<400 \mathrm{~m}$ ) better agreed field measurements than in steady inlet conditions. Realistic wind conditions improve LES prediction substantially. In far field (i.e. $>400 \mathrm{~m}$ ) improvements in LES prediction was marginal: discrepancies between LES and field data remains substantial.

Other factors not considered in Xie (2011) are likely to blame. Thermal stability over cities is weaker than rural environments because of greater friction velocity $u_{*}$ over cities - perhaps one reason few publications report modelling urban-stability effects on dispersion. This recently attracted more attention, such as thermal stratification and its effects on flows in two-dimensional street canyons 
(Liu et al., 2003; Louka et al., 2002; Li et al., 2010; Cai, 2012). Richards et al. (2006) and Boppana et al. (2012) investigated thermal effects near a heated cube in a deep surface layer over a rough wall. Kanda and Moriizumi (2009) studied momentum and heat transfer over a group of large blocks (COSMO experiments). Few LES papers exist of heat transfer over groups of blocks. Niceno and Hanjalić (2002) used LES to study heat transfer from a group of cubes with only one cube heated at very low Richardson number with negligible buoyancy effect, demonstrating LES as promising for heat-transfer applications but at cost: needing very fine near-wall resolution.

Using LES for flows and heat transfer at high Richardson number and Reynolds number $(\mathrm{Re})$ is challenging: accurate calculation for thin thermal layers on solid walls is required (Boppana et al., 2010). Resolving such layers for realistic Re is too expensive; appropriate thermal-wall models are not available yet. Therefore, firstly stratification effects on turbulent flows and dispersion of approach flows was investigated; building surfaces and ground were considered adiabatic walls. Local heat transfer from building and ground surfaces were ignored, and computing cost was saved. This was perhaps analogous to a well-developed non-neutral flow (e.g. through large urban areas or desert) approaching a neighborhood-scale region, where effects of local heat transfer was assumed less important.

Section 2 briefly presents the governing equations and numerical details, including geometry, mesh and boundary conditions. Section 3 presents LES results of flows under weakly non-neutral conditions over a group of staggered cubes: Case A. Section 4 presents comparison of LES data over DAPPLE site between neutral and weakly unstable conditions, and validation using wind-tunnel and field data: Case B. Conclusions and final remarks are presented in Section 5.

\section{Governing Equations and Numerical Settings}

\subsection{Governing Equations}

A brief description of governing equations is given here, more details for flow and scalar are in Xie and Castro (2006), hereafter XC. The filtered continuity and Navier-Stokes equations are,

$$
\begin{aligned}
& \frac{\partial \bar{u}_{i}}{\partial x_{i}}=0 \\
& \frac{\partial \bar{u}_{i}}{\partial t}+\frac{\partial \bar{u}_{i} \bar{u}_{j}}{\partial x_{j}}=-\frac{1}{\rho}\left(\frac{\partial \bar{p}}{\partial x_{i}}\right)+\frac{\partial}{\partial x_{j}}\left(\frac{\tau_{i j}}{\rho}+v \frac{\partial \bar{u}_{i}}{\partial x_{j}}\right) .
\end{aligned}
$$


The dynamical quantities, $\bar{u}_{i}, \bar{p}$ are resolved-scale (filtered) velocity and pressure respectively. $\rho$ and $v$ are respectively density and kinematic molecular viscosity. $\tau_{i j}$ is the subgrid-scale (SGS) Reynolds stress. The Smagorinsky SGS model was used with the constant $C_{s}=0.1$,

$$
\tau_{i j}-\delta_{i j} \tau_{k k} / 3=2 \rho\left(C_{s} \Delta\right)^{2}\left(2 \bar{s}_{m n} \bar{s}_{m n}\right)^{1 / 2} \bar{s}_{i j}
$$

where $\bar{s}_{i j}=\frac{1}{2}\left(\frac{\partial \bar{u}_{i}}{\partial x_{j}}+\frac{\partial \bar{u}_{j}}{\partial x_{i}}\right) ; \Delta$ is taken as the cube root of cell volume. $\delta_{i j}$ is the Kronecker-delta. In the near-wall region, the Lilly damping function was applied.

The filtered scalar and temperature transport equations are,

$$
\begin{gathered}
\frac{\partial \bar{c}}{\partial t}+\frac{\partial \bar{u}_{j} \bar{c}}{\partial x_{j}}=\frac{\partial}{\partial x_{j}}\left[\left(K_{s}+K_{m}\right) \frac{\partial \bar{c}}{\partial x_{j}}\right]+S \text { and } \\
\frac{\partial \bar{\theta}}{\partial t}+\frac{\partial \bar{u}_{j} \bar{\theta}}{\partial x_{j}}=\frac{\partial}{\partial x_{j}}\left[\left(d_{s}+d_{m}\right) \frac{\partial \bar{\theta}}{\partial x_{j}}\right],
\end{gathered}
$$

where $\bar{c}$ and $\bar{\theta}$ are respectively resolved-scale (filtered) scalar and absolute temperature. $S$ is the source term - a function of space and time. $K_{m}$ and $d_{m}$ are molecular diffusivity. $K_{s}$ and $d_{s}$ are the subgrid diffusivity, and are modelled using the subgrid eddy viscosity (Eq. 2) combined respectively with a constant unity subgrid-scale Schmidt number and with a constant subgrid-scale Prandtl number 0.9 , as usual.

The discretisation for all terms in Eqs. 1-4 was second-order accurate in both space and time. A second order monotone advection and reconstruction scheme (MARS) for the convective terms in space were applied to solve Eqs. 3 and 4. The MARS is used to capture sharp gradients at scalar plume's edge and to avoid spurious negative concentrations.

\subsection{Settings of inlet boundary conditions}

Appropriate settings of inlet boundary conditions are crucial for LES. However, field and wind-tunnel data are scarce. LES numerical experiments were inevitably necessary to test results' sensitivity to critical parameters. Since only weakly unstable or stable conditions were considered here, turbulence statistics used in Xie and Castro (2008) and Xie and Castro (2009) for inflow conditions which were fitted from neutral wind-tunnel measurements, were respectively used to generate inflow conditions for flows over a group of staggered cubes (Case A) in 


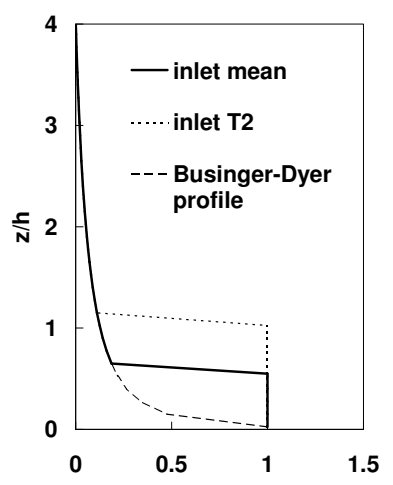

(a)

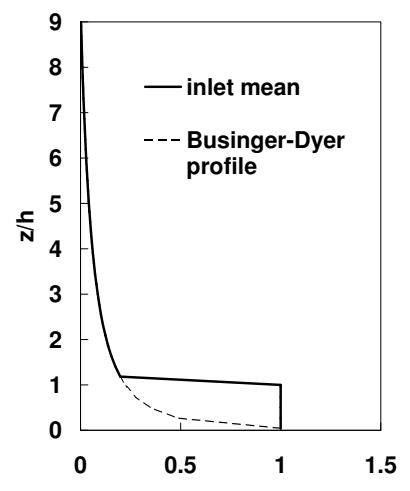

(b) $\quad\left(\theta-\theta_{\mathrm{a}}\right) /\left(\theta_{\mathrm{f}}-\theta_{\mathrm{a}}\right)$

Figure 1: Dimensionless mean temperature profiles at inlet. (a) for Case A; (b) for Case B. $\theta_{a}$, freestream temperature; $\theta_{f}$, ground floor temperature; h, mean building or cube height.

$\S 3$ - and flows and dispersion over DAPPLE site (Case B) in $\S 4$. Specific settings are in $\S 2.3$ and $\S 2.4$ respectively.

DAPPLE field experiments on 15/05/2003 (Martin et al., 2010a) have unavailable temperature data. Temperature profiles specified for Case B at inlet boundaries B1, B2 and B4 (see Fig.4) were approximated using Businger-Dyer relations for unstable conditions from BT Tower data on 03/06/2004 (Martin et al., 2010b; Wood et al., 2009). We focus on numerical experiments investigating thermalstability effects of approach flows by varying bulk Richardson number $R_{b}$, rather than estimating an 'accurate' one. $R_{b}$ is

$$
R_{b}=g \Delta \theta h /\left(\theta_{a} U_{r e f}^{2}\right),
$$

where $\Delta \theta$ is the difference between ground temperature $\theta_{f}$ and freestream temperature $\theta_{a} ; g$ is acceleration due to gravity; $h$ is average building height; $U_{r e f}$ is freestream velocity.

Fig. 1 shows dimensionless mean inlet temperature $\left(\theta-\theta_{a}\right) /\left(\theta_{f}-\theta_{a}\right)$. BusingerDyer profiles are derived from Businger-Dyer relations (Stull, 1988, pp360-361). The sharp peak in the near-wall region of the original Businger-Dyer profile decayed rapidly when converted from inlet into the domain without wall heating. We assumed that in the near-wall region (i.e. $z / h \leq 0.55$ ) the temperature was well mixed and was approximated as constant as shown in Fig. 1 (a) for Case A. In Fig. 1 (a) and (b) temperature was approximated as constant within canopy (i.e. $z / h \leq 1.0)$ as a numerical experiment for Cases A and B. 
Variances and integral length scales of temperature fluctuations are required for generation of instantaneous inlet temperature fluctuations. We followed the same procedure as that of turbulence generation in Xie and Castro (2008, 2009). Since temperature-fluctuation statistics were unavailable, it is crucial to know the effect of inlet temperature fluctuations on velocity fluctuations and mean velocity. Subsequently numerical experiments were conducted as follows.

(I) The instantaneous inlet temperature was

$$
\theta(y, z, t)=\bar{\theta}(z)
$$

where no temperature fluctuation was superimposed on the mean profile.

(II) Variance of inlet temperature fluctuations was approximated using empirical relationships in surface layers under weakly stratified conditions (Stull, 1988, pp366), i.e. $\theta_{r m s} / \theta_{*} \sim 2$, where $\theta_{*}=\overline{w^{\prime} \theta^{\prime}} / u_{*}$ is surface-layer temperature scaling parameter. Temperature fluctuations and heat fluxes measured atop BT Tower Westminster City Council House rooftop on 03/06/2004 (Wood et al., 2009) were used as baseline for the approximation; NB this is Case B data ( $\S 4)$. During 16:0017:00, $\theta_{r m s} / \theta_{*}$ was approximately 1.9 for rooftop data. A constant variance and same mean temperature profile as in (I) were used to produce instantaneous inlet temperature,

$$
\theta(y, z, t)=\bar{\theta}(z)+\theta^{\prime}(y, z, t),
$$

where $\theta^{\prime}$ integral length scales was set equivalent to those of wall-normal velocity.

(III) The instantaneous inlet temperatures were generated similarly as that in (II), except that integral length scales of $\theta^{\prime}$ were set equivalent to the averaged length scale of velocities $u, v$ and $w$.

\subsection{Specific settings for flows over a group of staggered cubes - Case A}

Flows and heat transfer over a group of cubes mounted on a wall provides a test case for validating urban LES (Boppana et al., 2010). Details of mean velocities, Reynolds stresses and turbulence integral length scales for Case A are in Xie and Castro (2008). LES with efficient inflow conditions was applied to calculate turbulent flows over a group of staggered wall-mounted cubes and was validated against neutral wind-tunnel experiments. Here a brief description of computational domain and boundary conditions is given.

Since only weakly thermal stratification was considered, turbulence statistics and integral length scales which were applied for generation of inflow data were assumed the same as those for neutral conditions in Xie and Castro (2008). This 
helps to isolate whether thermal stratification (or temperature gradient) impacts turbulence or dispersion. Parsimoniously, $U_{m}(z), \overline{u^{\prime} w^{\prime}}(\mathrm{z}), u_{r m s}(z), v_{r m s}(z), w_{r m s}(z)$ profiles were not functions of $y$. Although easily performed, in practical cases it is unlikely that sufficient knowledge of such spanwise variations would be available to make it either sensible or possible. Turbulence profiles approximated horizontally averaged measurements (Castro et al., 2006). They were simplified and thus differ from the 'real' values at some within-canopy locations, but the LES results were barely sensitive to these discrepancies.

$R e$ was approximately 3,000 based on freestream velocity and cube height. $R_{b}$ numbers $-0.2,-0.1,0,0.1$ and 0.2 of approach flows were investigated. Case A comprises eight rows of staggered cubes: four repeated units stacked in the streamwise direction (Fig. 2). Converged turbulence statistics were produced in such a domain (Xie and Castro, 2008). The four vertical lines indicate data sampling locations and are subsequently denoted (left-right) by 'behind row 1', 'behind row 3', 'behind row 5' and 'behind row 7'. These 4 stations all correspond to P1 station in Fig. 2b. Fig. 2b shows a plan view of one repeated unit of the staggered wall-mounted cube array. The domain height was $4 h$, with $h$ defined as cube height. P0, P1, P2 and P3 denote the four typical data-sampling locations. The synthetic inflow data was imposed at the inlet and zero-gradient outflow conditions at the outlet. At domain-top, stress-free conditions were applied. Periodic boundary conditions were used in lateral directions. Solid-wall boundary conditions with a wall model were applied for all other boundaries (details in XC). A uniform mesh of more than one million cells with $16 \times 16 \times 16$ grid points per cube was used, as suggested in XC for sufficient accuracy in these flow types.

\subsection{Specific settings for flows and dispersion over DAPPLE site-Case B}

Urban dispersion experiments in central London were conducted in the DAPPLE project (Wood et al., 2009). DAPPLE focussed on the intersecting Marylebone Road and Gloucester Place (Dobre et al., 2005; Balogun et al., 2010) using full-scale dispersion experiments and micro-meteorological data. DAPPLE's methods are that an inert and passive tracer gas is released from a fixed point, and the gas is sampled at stations in the near-field. Here, we use data from two field experiments. First, 15/05/2003 at 17:00 local time (Martin et al., 2010a). Second, 03/06/2004 at 16:30 local time (Martin et al., 2010b). The time-resolved experiments had a release for 15 minutes, concurrent with sampling for 30 minutes (i.e. continuing after release ends) of ten 3-minute samples.

Micro-meteorological measurements have operated almost continuously from 2004 to present (Wood et al., 2010) atop BT Tower. BT Tower is about $1.5 \mathrm{~km}$ east 


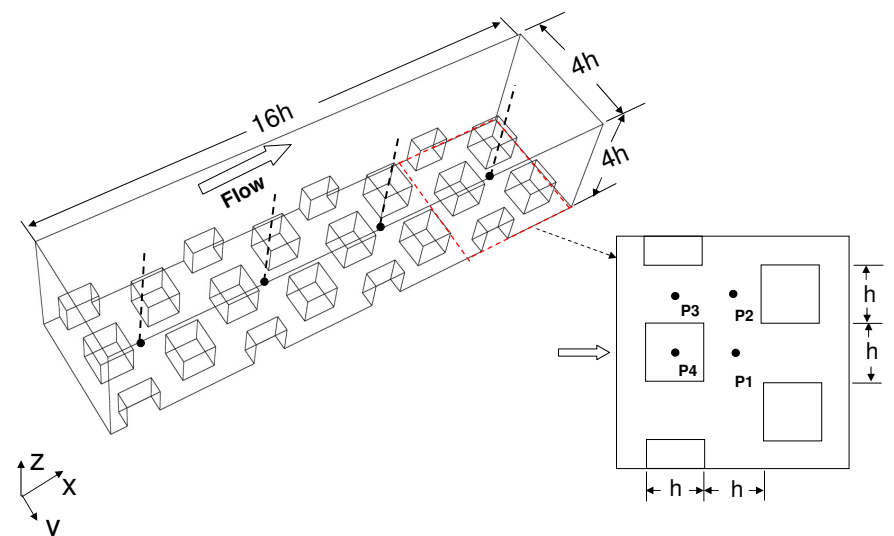

(a)

(b)

Figure 2: (a) Schematic view of the domain of a group of staggered cubes. (b) Plan view of one repeated unit. P1, P2, P3 and P4, four typical sampling stations.

Low resolution model (1:200)

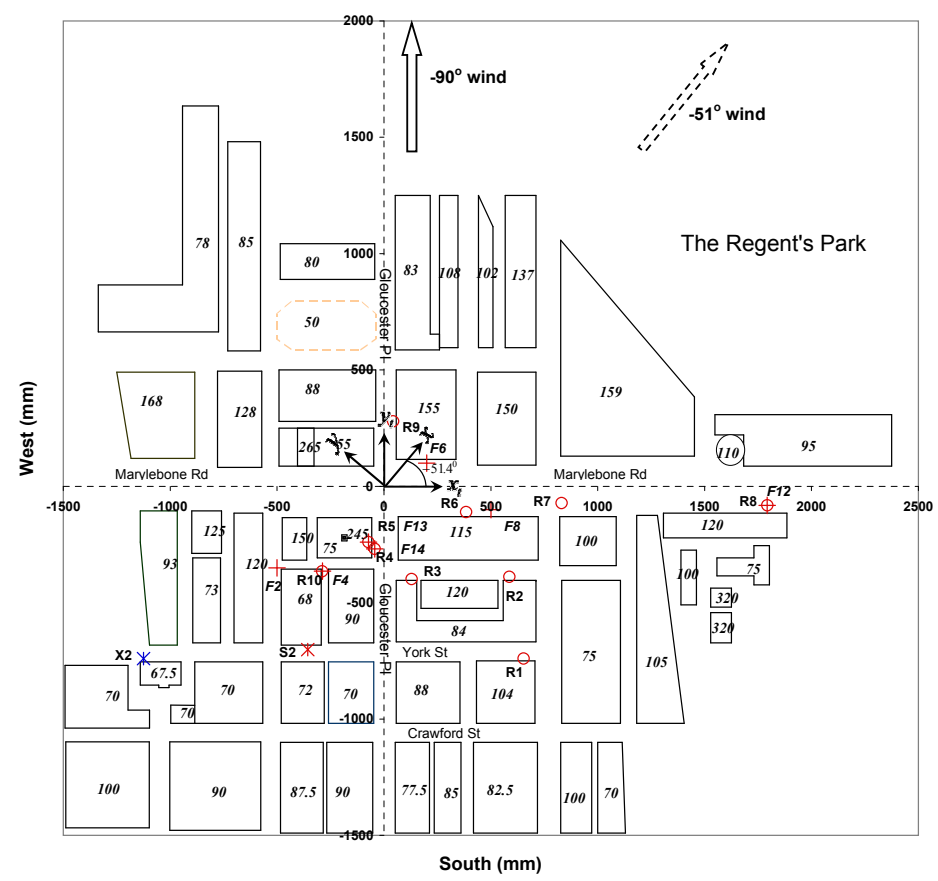

Figure 3: Wind-tunnel model plan view. Heights in mm (italics on blocks). $S 2$ and $X 2$, model and field sources respectively. $R 1-R 10$, sampling stations in steady winds ( $\$ 4.1) . F 2-F 14$, sampling stations in a realistic wind (§4.2). Model coordinates marked in mm, with $x_{t}$ from west to east, $y_{t}$ from south to north and $z$ from ground to top respectively. $x, y, z$, computational coordinates (Fig.4). 


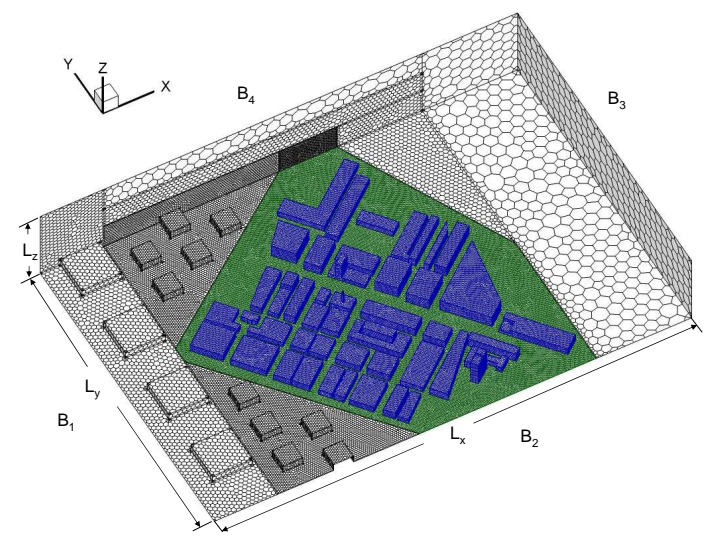

Figure 4: Computational domain with mesh. The coordinate origin at the ground, at the DAPPLE intersection. $L_{x}=6000 \mathrm{~mm}, L_{y}=4000 \mathrm{~mm}, L_{z}=1000 \mathrm{~mm}$ in model scale.

of the DAPPLE intersection. Equipment are mounted on a lattice mast atop the main tower, giving a measurement at $190.3 \mathrm{~m}$ above ground (Barlow et al., 2011); thus the measurements are about 9 times higher than mean building height in the DAPPLE area. A Gill R3-50 sonic anemometer gave $10 \mathrm{~Hz}$ 3D winds and ultrasonic temperature (approximately virtual temperature): fluxes of sensible heat and momentum can also be estimated from these data (for atmospheric stability estimation). Data were quality-controlled using standard micro-meteorological procedures (Wood et al., 2010). We took means (30-sec and 60-sec) of raw data.

$R_{b}$ during DAPPLE field experiments varied from -0.07 to -0.1 during 16:0017:00 on 03/06/2004; it varied from -0.05 to -0.17 during 12:00 - 20:00 (Wood et al., 2009). The $R_{b}$ was estimated based on the wind speeds and temperatures measured atop BT Tower and the 16-metre-high rooftop that was Westminster City Council House (adjacent to the DAPPLE intersection). A few numerical experiments (i.e. $R_{b}=-0.01,-0.03,-0.1$, without local heating) were performed. For using BT-Tower data, the LES was initialised at 16:00 on 03/06/2004. The nonreactive tracer was released at 16:30, when the sampling was started, and turned off at 16:45 - but sampling continued until 17:00, when instantaneous concentration was zero at all sites (Martin et al., 2010b).

We simulated flow and dispersion over a wind-tunnel model - the 1:200 lowresolution model. The numerical model's detailed description is in Xie and Castro (2009) and Xie (2011); a brief description is given here. Re, based on freestream velocity and mean building height of wind-tunnel model, was about 18,000. A model plan view is shown (Fig. 3). The arrows with solid line and dashed lines 
indicate $-90^{\circ}$ and $-51^{\circ}$ wind respectively. Wind direction is defined relative to the Marylebone-Road direction, i.e. $x_{t}$ in Fig. 3.

The domain size $L_{x}=6000 \mathrm{~mm}, L_{y}=4000 \mathrm{~mm}, L_{z}=1000 \mathrm{~mm}$ (see Fig. 4) is $1.2 \mathrm{~km}, 0.8 \mathrm{~km}$ and $0.2 \mathrm{~km}$ respectively in full scale. The finest resolution in the near wall region is $1 \mathrm{~m}$; total cell number is 1.3 million. Mean building-block height is $h=110 \mathrm{~mm}$; packing density is 0.5 . Except for a few tall buildings, one small tower and one dome, most of the buildings are nearly cuboid with low and differing heights. The building-block arrangement is mainly in staggered and aligned patterns with intersections and ' $T$ ' junctions. The street-canyon pattern seems more dominant for south-north streets than east-west streets.

Boundaries B1, B2 and B4 (see Fig. 4) were set as inlets for realistic winds. For $-51^{\circ}$ wind only the boundary B1 was set as an inlet, while B2 and B4 were set as symmetric walls. At inlet boundaries an inflow approach (Xie and Castro, 2008) with the same mean velocities, Reynolds stresses and integral length scales as in Xie and Castro (2009) and Xie (2011) was used to generate turbulence fluctuations correlated in space and in time. Boundary B3 was set as an outlet. The domain's upper boundary was set as a stress-free wall and other boundaries were set as solid walls. The wind directions and magnitudes on 03/06/2004 were used alike in Xie (2011). At every time step, we scaled the Reynolds stresses accordingly, based on mean velocity magnitude. More detailed descriptions are in Xie and Castro $(2008,2009)$. We generated turbulence fluctuations at a plane normal to wind direction in a local coordinate system based on the plane and then projected the velocities on the computational domain coordinate for inlet boundaries.

LES dispersion in the $-51^{\circ}$ and $-90^{\circ}$ winds was validated and reported in Xie and Castro (2009) and Xie (2011) using wind-tunnel data. Here, dispersion in the $-51^{\circ}$ and $-45^{\circ}$ winds in various thermal-stratification conditions was simulated and validated. Xie (2011) also reports LES of dispersion in realistic winds under neutral conditions. To investigate thermal-stratification effects in realistic winds, some numerical experiments are reported here.

\section{Results of flow over a group of staggered cubes - Case A}

\subsection{Effects of inlet temperature fluctuations}

Fig. $5(\mathrm{a}, \mathrm{b})$ shows turbulence statistics profiles at station $\mathrm{P} 1$ behind row 7 (see Fig. 2) of numerical experiments (I) and (II) at $R_{b}=-0.1$, and are compared with those in neutral conditions. The unstable thermal conditions enhance velocity fluctuations evidently. Fig. $5(\mathrm{a}, \mathrm{b})$ also shows that the effect of inlet temperature

fluctuations on velocity fluctuations, in particular $w_{r m s}$, is visible. However, the 

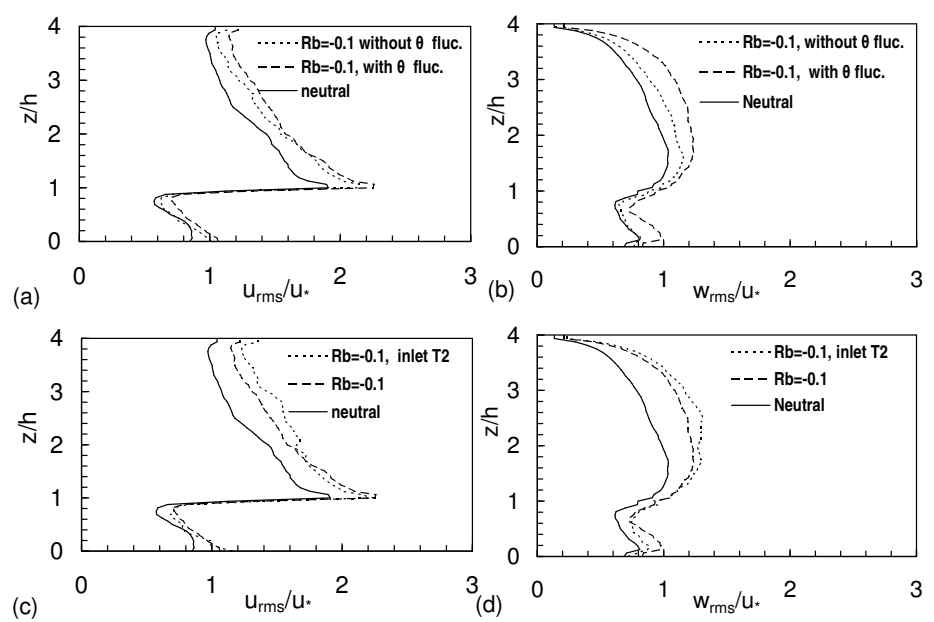

Figure 5: Sensitivity to inlet conditions. Effects of inlet temperature fluctuations (a, b) and shape of mean temperature profiles (c, d) on velocity fluctuation $r m s$ at station $P 1$ behind row 7 (see Fig. 2). $u_{*}$, mean friction velocity.

effects on the mean velocity profile seem very small (not shown). Further, the effect of integral length scales of inlet temperature fluctuations on velocity fluctuations and mean velocity at station P1 behind row 7 is unsubstantial, as long as reasonable length scales (e.g. in the order of the block size) were used. This is consistent with conclusions for turbulence length scales in Xie and Castro (2008).

We also check the effect on turbulence of the profile shape of the mean temperature at the inlet. Fig. 1(a) shows two dimensionless-mean-temperature profiles specified at the inlet. Fig. 5(c,d) shows the stability effect of the shape of the mean temperature profile at the inlet on the velocity fluctuations at station $P 1$ behind row 7. The effect on velocity fluctuation $r m s$ is visible but is relatively small, whereas the effect on mean velocity is hardly discerned (not shown).

\subsection{Effects of thermal stratification}

Fig. 6 shows stability effects of approach flows on mean velocity vectors $(U, W)$ on a vertical plane at $\mathrm{P} 1$ behind row 5. Fig. 6(a) (unstable) shows a larger circulation region in front of the cube than those in (b)(stable) and (c)(neutral). The effect of the stable thermal condition on flows over staggered cubes are quite different from those for street canyons (e.g. Louka et al., 2002). Perhaps this is due to flows being highly three dimensional and scales of eddies within and immediately above the canopy being dominated by cube size. In the unstable condition (i.e. $R_{b}=-0.2$ ), the velocity magnitude immediately behind cube was 


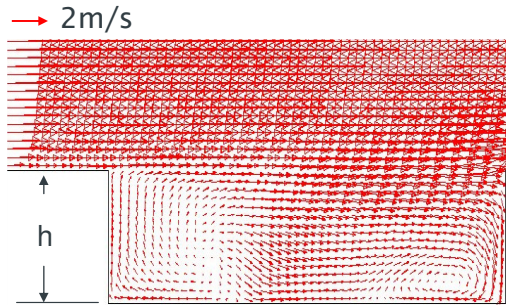

(a) $\mathrm{Rb}=-0.2$ (unstable)

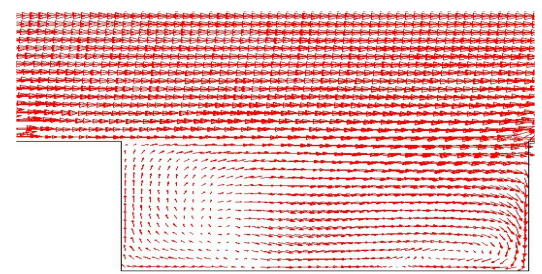

(c) $\mathrm{Rb}=0$ (neutral)

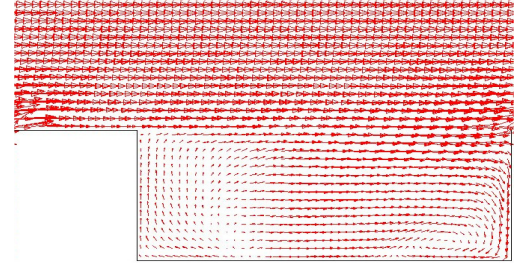

(b) $\mathrm{Rb}=0.2$ (stable)

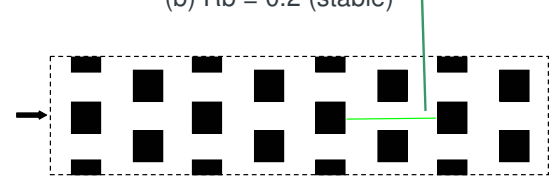

(d) Plan view of geometry

Figure 6: Mean velocity vectors (UW) on a vertical plane marked in (d) under various stratification conditions. (a) $R_{b}=-0.2$; (b) $R_{b}=0.2$ and (c) $R_{b}=0$.(d) domain's plan view.

slightly greater than those for $R_{b}=0$. In contrast, the difference of mean flow field between Fig. 6(b) and (c) is hard to discern.

Mean velocity vectors $(U, V)$ on a horizontal plane at half cube height show slightly stronger two counter circulations behind the cube under $R_{b}=-0.2$ than those under $R_{b}=0.2$ and 0 . This is consistent with Fig. 6 which shows that the unstable condition enhances the recirculation bubble in front of the cube.

Fig. 7 shows velocity fluctuation $r m s$ and mean velocity at station $P 1$ behind row 7 , under various stratification conditions, i.e. $R_{b}=-0.2,-0.1,0,0.1$ and 0.2 . The turbulent fluctuation fields in unstable conditions differ evidently from that in the neutral condition. However, the fluctuation fields in stable conditions show only small difference compared with those in the neutral condition. Again, this is probably due to turbulent flows being block-size dominant during weakly stable and neutral conditions. And buoyancy is less effective to suppress turbulent motions than that in two-dimensional street canyon flows. The mean flow fields do not change evidently with $R_{b}$.

\subsection{Domain-size effects}

The computational domain height in unstable conditions is more a concern than in neutral or stable conditions. A larger domain, with height $10 \mathrm{~h}$, but other 


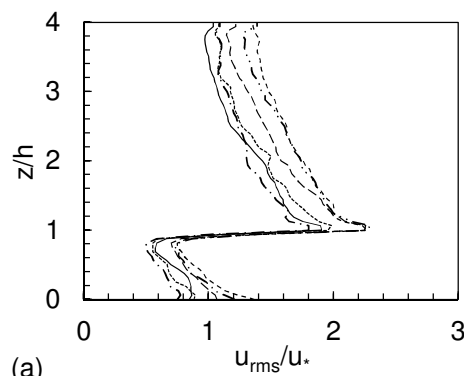

(a)
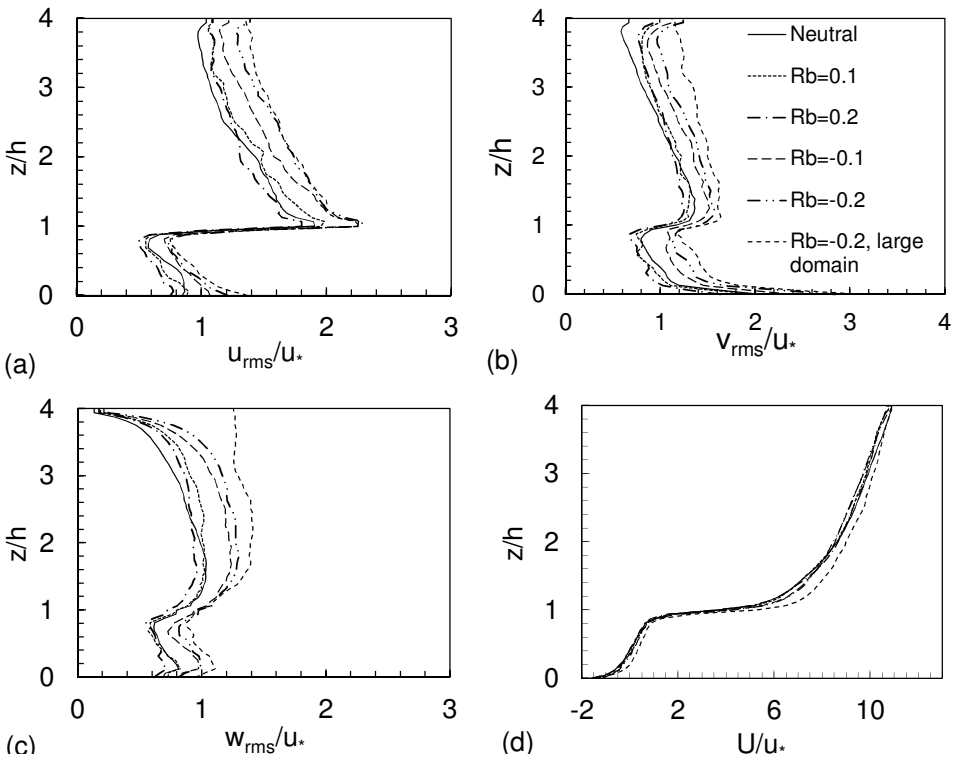

Figure 7: Velocity fluctuation $r m s$ and mean velocity at station $P 1$ behind row 7 , under stratification conditions $R_{b}=-0.2,-0.1,0,0.1$ and 0.2. 'large domain', domain height $10 \mathrm{~h}$.

settings same as those in Fig. 2 was designed to test domain-size effects. Fig. 7 shows that velocity fluctuation $r m s$ and mean velocity at $R_{b}=-0.2$ for the larger domain are barely greater than those for the smaller domain - a small domain height $4 h$ barely affects results. Hanna et al. (2002) suggest that at stations behind row 7 (i.e. $x \sim 14 h$ ), the flow field is fully converged. We noticed that at $x \sim 14 h$, the temperature field seemed converged too.

\section{Results of dispersion over DAPPLE site-Case B}

\subsection{In steady winds}

LES and wind-tunnel experiments were performed to investigate effects of thermal stratification on flow and dispersion over the DAPPLE site in steady $-51^{\circ}$ and $-45^{\circ}$ winds. No temperature fluctuation was superimposed on mean temperature at LES inlet.

Fig. 8 shows instantaneous temperature contours on a vertical $(x-z$, at $y=$ $0)$ plane crossing the DAPPLE intersection in unstable $\left(R_{b}=-0.2\right)$ and stable $\left(R_{b}=0.2\right)$ conditions in the $-51^{\circ}$ wind. The distance from inlet to the DAPPLE intersection is $27 \mathrm{~h}$ : sufficient for flow and temperature fields to be fully developed 


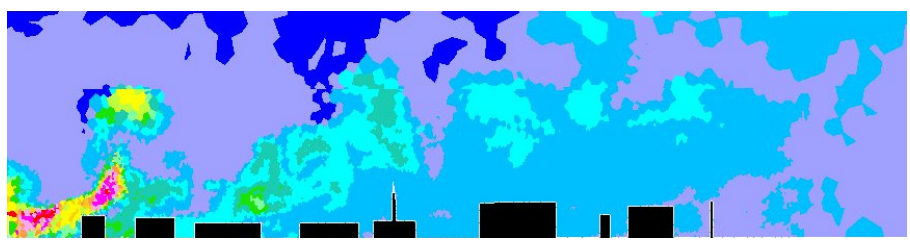

(a) $R_{b}=-0.2$

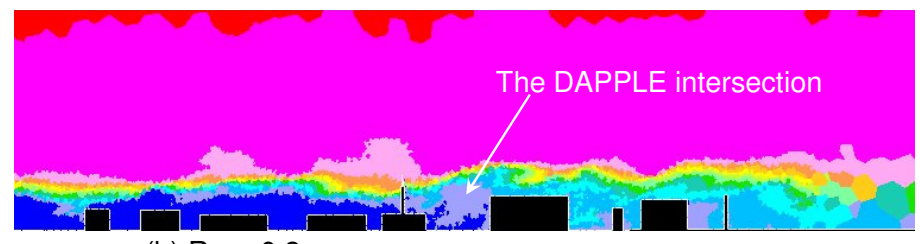

(b) $R_{b}=0.2$

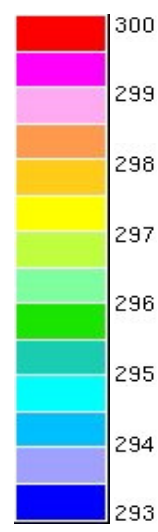

293

Figure 8: Instantaneous temperature contours $(\mathrm{K})$ on a vertical $(x-z$ at $y=0)$ plane crossing the DAPPLE intersection in the $-51^{\circ}$ wind. Wind direction is from left to right. (a) $R_{b}=-0.2 ;$ (b) $R_{b}$ $=0.2$.

within and immediately above canopy ( 33 ). This is confirmed again in Fig. 8. And the temperature field converges earlier in the stable condition (Fig. 8b) than the unstable condition (Fig. 8a).

For wind-tunnel experiments and LES, a non-reactive tracer was released from a steady ground-level point source $S 2$ (Fig. 3) in some stratification conditions; in field experiments the release duration was 15 minutes in one stratification condition. Fig. 9 shows comparison of dimensionless mean concentration along Marylebone Road and Gloucester Place under various thermal stratification conditions between wind-tunnel and LES data. A sensitivity test of dispersion to wind directions was performed: dispersion is not insensitive to wind directions under non-neutral conditions, consistent with that under a neutral condition Xie (2011). Fig. 9 shows that case 'LES, $R_{b}=-0.2,-45^{\circ}$ ' is generally in better agreement with the measurements 'wind tunnel, $R_{b}=-0.2,-45^{\circ}$ ' than case 'LES, $R_{b}$ $=-0.1,-45^{\circ}$. This might suggest that the temperature inlet boundary conditions of LES are reasonable, even though inlet temperature settings were not exactly identical to those in wind-tunnel experiments. The mean concentration of case 'LES, $R_{b}=0.2,-51^{\circ}$ ' differs greatly with measurements 'wind tunnel, $R_{b}=0.04$, $-51^{\circ}$ : unsurprisingly, dispersion is sensitive to thermal stratification. Fig. 9 (a) shows almost-constant mean concentration downstream of the DAPPLE intersection $\left(x_{t}>0\right)$ : due to channelling in Marylebone Road. A substantial concentration 

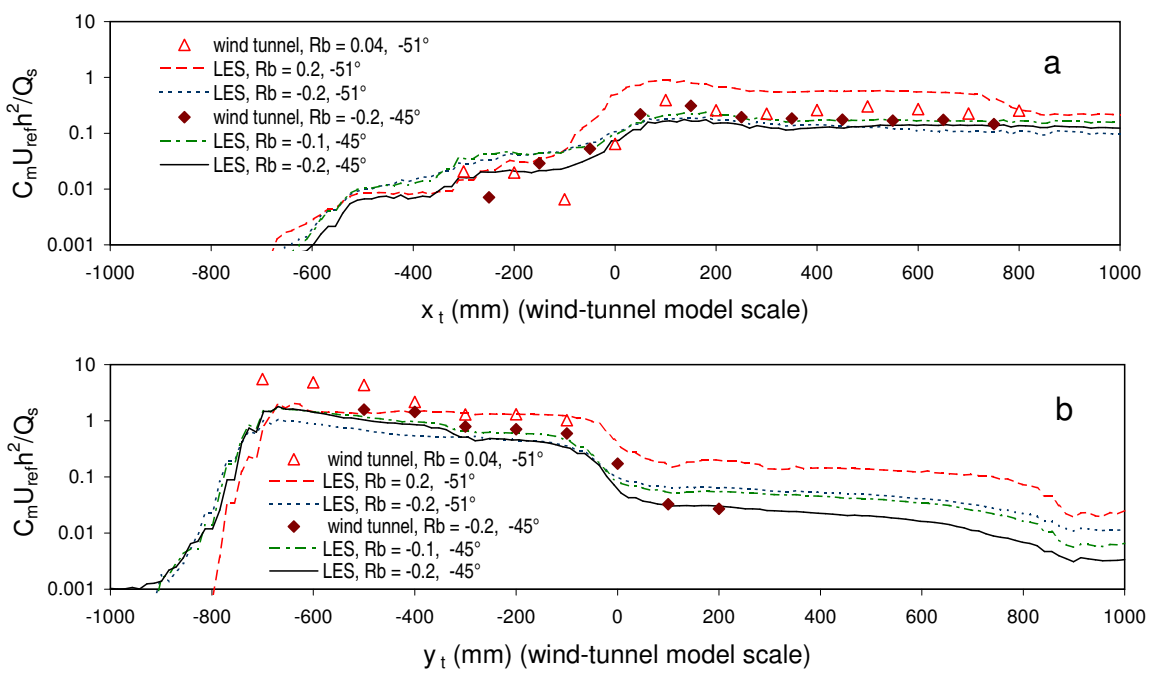

Figure 9: Non-dimensionalised mean concentration at height $z / h_{m}=0.1$ along (a) Marylebone Road $\left(y_{t}=0\right)$ and (b) Gloucester Place $\left(x_{t}=0\right)$ in various thermal stabilities. $U_{\text {ref }}$, free-stream velocity. $Q_{s}$, source concentration flux. $h$, mean building height. Abscissa in wind-tunnel model scale. 


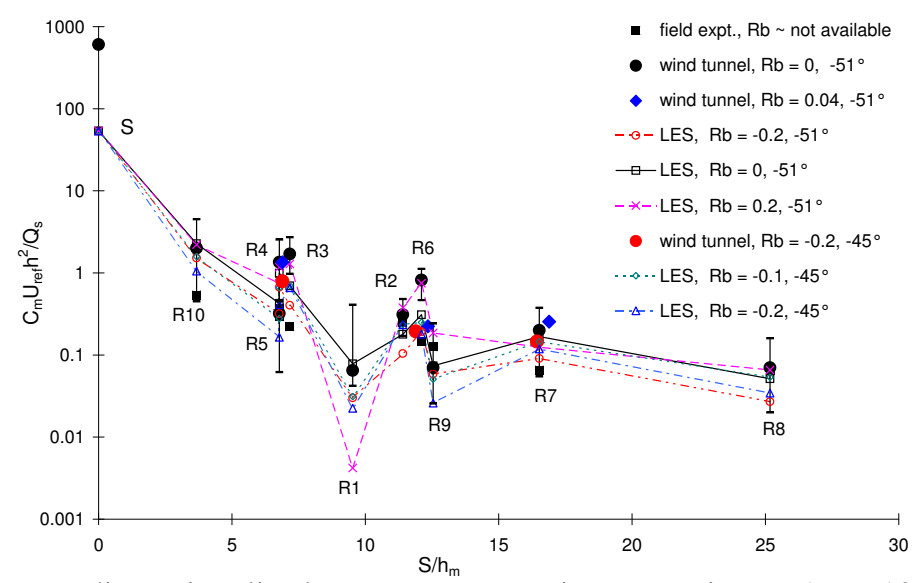

Figure 10: Non-dimensionalised mean concentration at stations $R 1-R 10$ in various thermal stabilities. $U_{\text {ref }}$, free-stream velocity. $Q_{s}$, source concentration flux. $h$, mean building height. $S=\left|x_{R}-x_{S}\right|+\left|y_{R}-y_{S}\right|$, street distance to source, where $\left(x_{R}, y_{R}\right)$ and $\left(x_{S}, y_{S}\right)$ are site and source coordinates respectively.

drop in Fig. 9 (b) and substantial increase in Fig. 9 (a) downstream of the DAPPLE intersection $\left(x_{t}, y_{t}=0\right)$ under all stratification conditions suggest that thermal buoyancy does not affect the major path (i.e. Gloucester Place - the DAPPLE intersection - Marylebone Road) of scalar convection in these two winds.

Fig. 10 shows comparison of dimensionless mean concentration at stations $R 1-R 10$. Field data were the maximum of 3-min bag concentration (Cheng and Robins, 2004). The concentration at site $R 1$ varied dramatically at different $R_{b}$ numbers, which was because $R 1$ was located at plume edge (see Fig. 3 ). The comparison between LES and corresponding wind-tunnel data for matching $R_{b}$ and wind direction is promising. Recall that compared with field measurements, LES using the realistic wind conditions in neutral conditions improved predictions for near field, but barely improved predictions for far field (Xie, 2011). It was suggested to include thermal stratification in LES. Lacking temperature measurements prevented estimation of $R_{b}$ for the 15/05/2003 field experiments in Fig. 10. Generally, wind-tunnel and LES data under weakly unstable conditions better agree with field data than those under neutral or weakly stable conditions. This may suggest that field experiments were conducted in a weakly unstable condition similar as that (e.g. $\left.R_{b} \sim-0.1\right)$ in $\S 4.2$. 


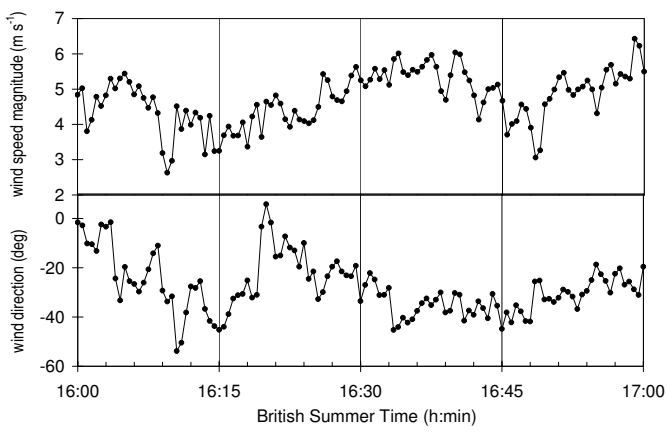

Figure 11: 30-sec averaged wind-speed magnitude and direction (i.e. bearing clockwise to Marylebone Road direction) at BT Tower on 03/06/2004. The source released from 16:30 to 16:45.

\subsection{In a realistic wind}

Real winds are never steady. We found that it was difficult to use weather data from operational numerical-weather-prediction models (e.g. the UK MetOffice's Unified Model) as boundary conditions to drive the street-scale LES (Xie, 2011). So we tried measured data with high temporal resolution. Fig. 11 plots 30- sec averaged horizontal wind velocity from 16:00-17:00 on 03/06/2004, which drove LES. Fig. 3 also shows source location $X 2$ and sampling stations $F 2, F 4, F 6, F 8$, $F 12, F 13$ and $F 14$ of DAPPLE field experiments on 03/06/2004. The LES was initialized at 16:00 with source release from 16:30 until 16:45, and with sampling and averaging from 16:30 until 17:00. This takes about 12 hrs (wallclock time) using 200 processors on a parallel computer.

Fig. 12 plots field measurements and six sets of LES results of 3-min averaged concentration at site $F 14$ (the Westminster City Council House doorway to the west of Gloucester Place, by the DAPPLE intersection) under four stratification conditions (i.e. $R_{b}=0,-0.01,-0.03,-0.1$ ). To check the sensitivity of initial conditions on LES results, two LES runs (i.e. 'LES, Rb=0,run 1' and ' $\mathrm{LES}, \mathrm{Rb}=0$,run 2') under a neutral condition were initialised from different conditions. Fig. 12 shows the effect of the initial conditions on the 3-min averaged concentration as unsubstantial. Under the same stratification condition $R_{b}=-0.01$, the results with inlet temperature fluctuations specified were in good agreement with those with no inlet temperature fluctuations, which confirms observations in $\S 3$. Hence, inlet temperature in other LES runs for the stratification conditions $R_{b}$ $=-0.03$ and -0.1 were set without fluctuations. Unsurprisingly, 3-min averaged concentration decreases with the decrease of $R_{b}$. LES with $R_{b}=-0.1$ produced evidently less concentrations than the measurements, which suggests that speci- 


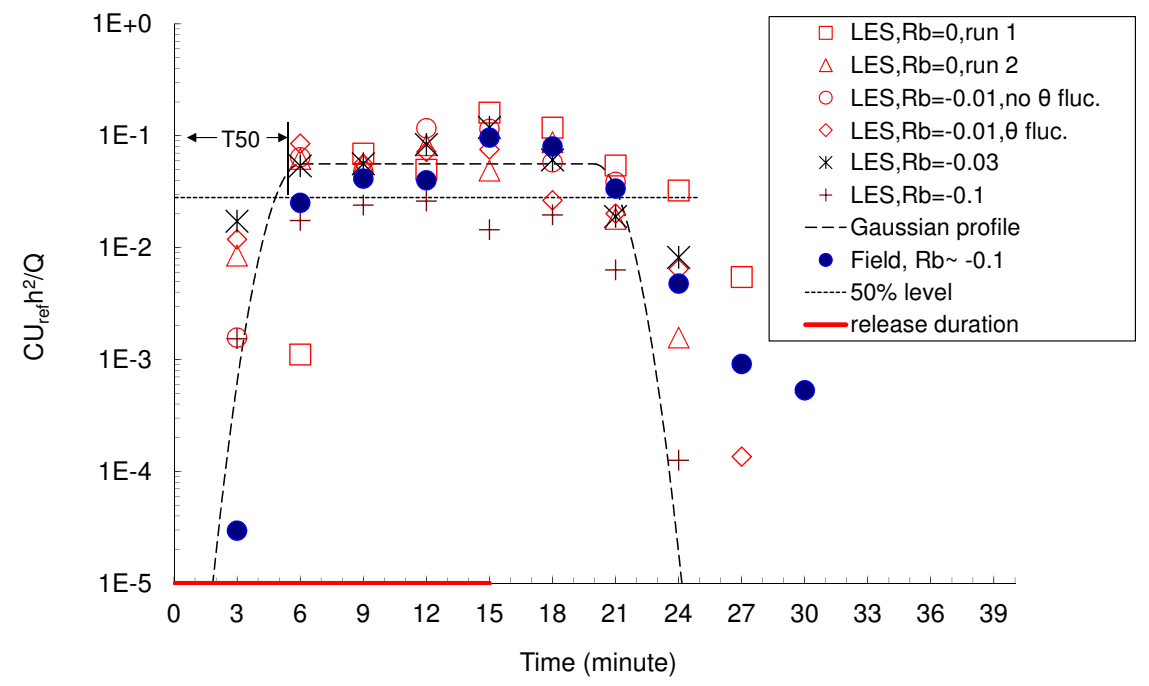

Figure 12: 3-min averaged concentration at site $F 14$ under various stratification conditions. $U_{\text {ref }}$, free-stream velocity; $Q$, source concentration flux; $h$, mean building height; red solid line: 15 minutes release duration; dashed line: approximate location and width of a plume in combined Gaussian form; dot line: 50\% level of maximum of the Gaussian profile; T50, approximate advection time.

fied $R_{b}=-0.1$ might be a less than the 'real' one in field experiments, although we estimated that $R_{b}$ was approximately -0.1 at $16: 00$ on $03 / 06 / 2004$.

LES results for $R_{b}=-0.03$ and -0.01 agree marginally better with measurements than those for $R_{b}=0$. The 'LES, $R_{b}=0$, run1' and 'LES, $R_{b}=0$, run2' data were fitted into a combined profile with two symmetric 'half Gaussian profiles' at the left and right end and a constant profile in the middle, where the constant is the maximum of the Gaussian profiles. The fitting was unideal because of the small available dataset, uncertainty and data asymmetry at the left and right ends of the profiles. However, the deduced time-scale and advection velocity are not too dependent on the quality of fitting as shown in Fig.12. The advection velocity of plume was estimated using

$$
U_{a d v}=\frac{D}{T 50},
$$

where $D$ is distance from site $F 14$ to source location $X 2, T 50$ is elapsed time (since release) when ensemble-averaged concentration reaches $50 \%$ its local maximum at Site $F 14$ (Cheng and Robins, 2004). Here T50 was estimated at approx- 


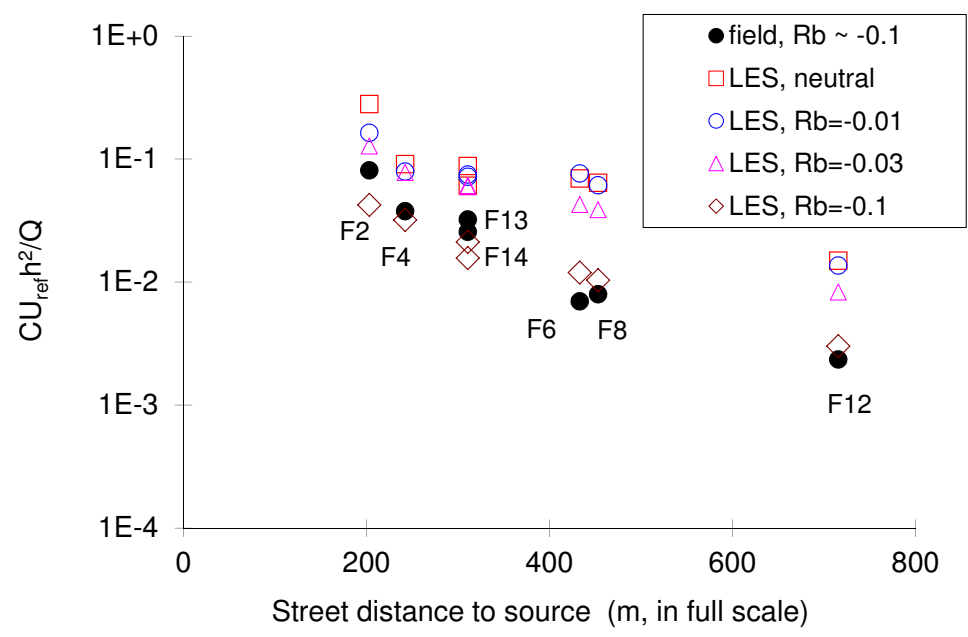

Figure 13: 30-min averaged concentration at sites $F 2, F 4, F 6, F 8, F 12, F 13$ and $F 14$. $U_{\text {ref }}$, freestream velocity; $Q$, concentration flux at source. $h$, mean building height. Street distance defined as $S=\left|x_{F}-x_{S}\right|+\left|y_{F}-y_{S}\right|$, where $\left(x_{F}, y_{F}\right)$ and $\left(x_{S}, y_{S}\right)$ are site and source coordinates respectively.

imately 5 minutes and advection velocity $U_{a d v}$ is about $0.15 U_{\text {ref }}$, which is close to $0.16 U_{\text {ref }}$ suggested in Cheng and Robins (2004), who performed wind-tunnel experiments in steady and neutral wind conditions. In Xie (2011) it is suggested that advection velocity in varying wind is similar to that in steady wind. Fig. 12 suggests that $T 50$ under weakly unstable conditions approximates that in neutral conditions.

Fig. 13 presents field measurements and LES results of 30-minute averaged concentration at the ' $F$ ' sites. The averaging was from 16:30 (since release) until 17:00. LES data for ' $R_{b}=-0.03$ ' are evidently less than those for 'neutral', but still greater than field measurements, in particular at far field. LES data for ' $R_{b}=$ -0.1 ' are substantially improved and overall best agree with field measurements at the 7 sites. However, in near field, i.e. $F 2, F 4, F 13$ and $F 14$, LES slightly under-predicted the 30-minute averaged concentration: consistent with Fig. 12. Perhaps it is impossible to generate LES data in 'good' agreement at all sites with measurements by tuning only $R_{b}$. So, if we consider that LES for $R_{b}=$ -0.1 is most appropriate, then we conclude that these settings account for farfield concentration, but differ at near field. This might be attributed to missing local heat transfer from buildings and ground surfaces or effects of small-scale roughness in LES model, to be tested in future work.

Overall, the effect of weakly unstable stratification on dispersion is not small. 
We also checked the 3D averaged concentration field. The concentration contours at a horizontal ground-level plane and at a vertical plane crossing the source respectively show greater upwind and lateral spreading and vertical spreading of the plume in weakly unstable stratifications than in the neutral condition. However, these numerical sensitivity experiments should be interpreted with caution. We assumed constant temperature within canopy. To reduce uncertainty due to the assumption, we presented LES data with various $R_{b}$ numbers. Nevertheless, we were unable to disregard the discrepancy between LES results and field measurements at all sites. Hence, perhaps more should be included in LES model, e.g. local thermal effects due to heat transfer through building and ground surfaces and effects due to small roughness elements.

\section{Conclusions}

Effects of thermal stratification on dispersion in urban areas were investigated using large-eddy simulations (LES), wind-tunnel and field experiments. This is a further study, since we previously considered effects of inflow turbulence and weather-scale wind variation on dispersion in neutral conditions (Xie and Castro, 2009; Xie, 2011). First, flows over a group of staggered cubes under some thermal stratifications, i.e. $R_{b}=0.2,0.1,0,-0.1$ and -0.2 were simulated. We found (1) turbulent fluctuations and mean velocities were not affected substantially either by a change of mean temperature profile below canopy height or inlet temperature fluctuations at matching $R_{b}$; (2) effects on the flow field of weakly unstable stratification conditions exceed those of weakly stable conditions at matching absolute $R_{b}$ for an array of staggered blocks. This was probably because under weakly stable conditions flows were fully three-dimensional and block-size scale was as dominant as that in neutral conditions.

Second, after some numerical experiments using the above simpler model, we studied thermal stratification effects of approach flows on turbulence and dispersion at DAPPLE site, central London. Based on estimated $R_{b}\left(-0.17 \leq R_{b} \leq\right.$ -0.05 ) from field data, a few $R_{b}$ based on average building height and freestream velocity, i.e. $-0.2 \leq R_{b} \leq 0.2$, of approach flows were chosen for numerical experiments with adiabatic boundary conditions at ground and building surfaces. We found (1) under these weak stratification conditions, mean concentration at certain stations can be up to one order different from that in steady or varying winds in neutral conditions; (2) LES under unstable conditions substantially improve numerical predictions of dispersion compared with that in neutral conditions. 
Stratification effects on dispersion even if under weakly unstable conditions in urban environments (e.g. in London) are not negligible. However, we were not able to disregard discrepancies between LES and field experiments in near nor far fields, by just tuning Richardson number. This suggests that more, e.g. effects of local heat transfer or small-scale roughness elements, should be included in the LES model for more-accurate simulations.

Acknowledgements This work is supported by NERC through NCAS, Grant R8/H12/38. We thank Profs Ian Castro, Alan Robins, Stephen Belcher and Janet Barlow, and Dr Bharathi Boppana for helpful comments. We thank Dr Fred Mendonca at CD-adapco for continuous support using Star-CD. Meteorological equipment were operated under projects DAPPLE, CityFlux and REPARTEE. Computations were performed on Iridis3 at University of Southampton, and HECToR at UK's supercomputer centre.

\section{References}

Balogun, A., Tomlin, A., Wood, C., Barlow, J., Belcher, S., Smalley, R., Lingard, J., Arnold, S., Dobre, A., Robins, A., Martin, D., Shallcross, D., 2010. Instreet wind direction variability in the vicinity of a busy intersection in central London. Bound.-Layer Meteorol. 136, 489-513.

Barlow, J., Harrison, J., Robins, A., Wood, C., 2011. A wind-tunnel study of flow distortion at a meteorological sensor on top of the BT Tower, London, UK. J. Wind Eng. Ind. Aerodyn. 99, 899-907.

Boppana, V.B.L., Xie, Z.T., Castro, I.P., 2010. Large-eddy simulation of dispersion from surface sources in arrays of obstacles. Bound.-Layer Meteorol. 135, 433-454.

Boppana, V.B.L., Xie, Z.T., Castro, I.P., 2012. Large-eddy simulation of heat transfer from a single cube mounted on a very rough wall. Bound.-Layer Meteorol. in press: DOI 10.1007/s10546-012-9793-7.

Cai, X.M., 2012. Effects of differential wall heating in street canyons on dispersion and ventilation characteristics of a passive scalar. Atmos. Environ. 51, 268 $-277$.

Castro, I.P., Cheng, H., Reynolds, R., 2006. Turbulence over urban-type roughness:decutions from with tunnel measurements. Bound.-Layer Meteorol. 118, 109-131. 
Cheng, H., Robins, A.G., 2004. Wind tunnel simulation of field tracer release in London, in: Zhuang, F.G., Li, J.C. (Eds.), Recent Advances Fluid Mech. Tsinghua Uni. Press \& Springer-Verlag, pp. 801-804.

Dobre, A., Arnold, S.J., Smalley, R.J., Boddy, J.W.D., Barlow, J.F., Tomlin, A.S., Belcher, S.E., 2005. Flow field measurements in the proximity of an urban intersection in London, UK. Atmos. Environ. 39, 4647-4657.

Gousseau, P., Blocken, B., Stathopoulos, T., van Heijst, G., 2011. CFD simulation of near-field pollutant dispersion on a high-resolution grid: A case study by les and rans for a building group in downtown Montreal. Atmos. Environ. 45, 428 $-438$.

Hanna, S.R., Tehranian, S., Carissimo, B., Macdonald, R.W., Lohner, R., 2002. Comparisons of model simulations with observations of mean flow and turbulence within simple obstacle arrays. Atmos. Environ. 36, 5067-5079.

Kanda, M., Moriizumi, T., 2009. Momentum and heat transfer over urban-like surfaces. Bound.-Layer Meteorol. 131, 385-401.

Li, X.X., Britter, R.E., Koh, T.Y., Norford, L.K., Liu, C.H., Entekhabi, D., Leung, D.Y.C., 2010. Large-eddy simulation of flow and pollutant transport in urban street canyons with ground heating. Bound.-Layer Meteorol 137, 187 - 204.

Liu, H.Z., Liang, B., Zhu, F.R., Zhang, B.Y., Sang, J.G., 2003. A laboratory model for the flow in urban street canyons induced by bottom heating. Adv. Atmos. Sci. 20, 554-64.

Louka, P., Vachon, G., Sini, J.F., Mestayer, P.G., Rosant, J.M., 2002. Thermal effects on the airflow in a street canyon-Nantes'99 experimental results and model simulations. Water, Air, Soil Pollution: Focus 2, 351-364.

Martin, D., Nickless, G., Price, C., Britter, R., Neophytou, M., Cheng, H., Robins, A., Dobre, A., Belcher, S., Tomlin, J.B.A., Smalley, R., Tate, J., Colvile, R., Arnold, S., Shallcross, D., 2010a. Urban tracer dispersion experiments in London (DAPPLE) 2003: Field studies and comparisons with empirical prediction. Atmos. Sci. Lett. 11, 241-248.

Martin, D., Price, C., White, I., Nickless, G., Petersson, F., Britter, R., Robins, A., Belcher, S., Barlow, J., Neophytou, M., Arnold, S., Tomlin, A., Smalley, R., 
Shallcross, D., 2010b. Urban tracer dispersion experiments during the second DAPPLE field campaign in London 2004. Atmos. Environ. 44, 3043-3052.

Niceno, B., Hanjalić, K., 2002. Turbulent heat transfer from a multi-layered wallmounted cube matrix: A large eddy simulation. Int. J. Heat Fluid Flow 23, 173-185.

Richards, K., Schatzmann, M., Leitl, B., 2006. Wind tunnel experiments modelling the thermal effects within the vicinity of a single block building with leeward wall heating. J. Wind Eng. Ind. Aerodyn. 94, 621-636.

Stull, R.B., 1988. An Introduction to Boundary Layer Meteorology. Kluwer Academic, the Netherlands.

Wood, C.R., Barlow, J.F., Belcher, S.E., Dobre, A., Arnold, S.J., Balogun, A.A., Lingard, J.J.N., Smalley, R.J., Tate, J.E., Tomlin, A.S., Britter, R.E., Cheng, H., Martin, D., Petersson, F.K., Shallcross, D.E., White, I.R., Neophytou, M.K., Robins, A.G., 2009. Dispersion experiments in central London: The 2007 DAPPLE project. Bull. Amer. Meteor. Soc. 90, 955-969.

Wood, C.R., Lacser, A., Barlow, J.F., Padhra, A., Belcher, S.E., Nemitz, E., Helfter, C., Famulari, D., Grimmond, C.S.B., 2010. Turbulent flow at 190 metres above London during 2006-2008: a climatology and the applicability of similarity theory. Bound.-Layer Meterol. 137, 77-96.

Xie, Z.T., 2011. Modelling of street-scale flows and dispersion in realistic winds - towards coupling with mesoscale meteorological models. Bound.-Layer Meteorol 141, 53-75.

Xie, Z.T., Castro, I.P., 2006. LES and RANS for turbulent flow over arrays of wall-mounted cubes. Flow, Turbul. Combust. 76, 291-312.

Xie, Z.T., Castro, I.P., 2008. Efficient generation of inflow conditions for largeeddy simulation of street-scale flows. Flow,Turbul. Combust. 81, 449-470.

Xie, Z.T., Castro, I.P., 2009. Large-eddy simulation for flow and dispersion in urban streets. Atmos. Environ. 43, 2174-2185. 